\title{
Convergence to global consensus in opinion dynamics under a nonlinear voter model
}

\author{
Han-Xin Yang, 1,2 , 6 Wen-Xu Wang, ${ }^{3}$ Ying-Cheng Lai, ${ }^{3,4}$ and Bing-Hong Wang ${ }^{2,5}$ \\ ${ }^{1}$ Department of Physics, Fuzhou University, Fuzhou 350002, P. R. China \\ ${ }^{2}$ Department of Modern Physics, University of Science and Technology of China, Hefei 230026, China \\ ${ }^{3}$ School of Electrical, Computer and Energy Engineering, Arizona State University, Tempe, AZ 85287 \\ ${ }^{4}$ Department of Physics, Arizona State University, Tempe, AZ 85287 \\ ${ }^{5}$ Research Center for Complex System Science, University of Shanghai for Science \\ and Technology and Shanghai Academy of System Science, Shanghai, 200093 China
}

(Dated: November 4, 2018)

\begin{abstract}
We propose a nonlinear voter model to study the emergence of global consensus in opinion dynamics. In our model, agent $i$ agrees with one of binary opinions with the probability that is a power function of the number of agents holding this opinion among agent $i$ and its nearest neighbors, where an adjustable parameter $\alpha$ controls the effect of herd behavior on consensus. We find that there exists an optimal value of $\alpha$ leading to the fastest consensus for lattices, random graphs, small-world networks and scale-free networks. Qualitative insights are obtained by examining the spatiotemporal evolution of the opinion clusters.

PACS numbers: 89.75.Hc, 87.23.Ge
\end{abstract}

\section{INTRODUCTION}

Mutual agreement, or consensus, is a fundamental phenomenon in social and natural systems. The dynamics of opinion sharing and competing and the emergence of consensus have become an active topic of recent research in statistical and nonlinear physics [1]. For example, a number of models have been proposed to address how consensus can result from the evolution of two competing opinions in a population, which include the voter model [2, 3], the majority rule model [4, 5], the bounded-confidence model [6] and the social impact model [7]. Due to the high relevance of complex networks [8] to social and natural systems, opinion dynamics have also been incorporated on networks [9-36] such as regular lattices, random graphs [42], small-world networks [43] and scale-free networks [44].

Previous works have revealed phase transitions in opinion dynamics [9, 10, 13, 15, 16] and the emergence of global consensus [3, 5] where all agents share the same opinion. It has also been found that both the network structures [12] and the opinion updating strategies [14, 17, 18] can affect the time for reaching the final consensus. In Ref. [37], Yang et al. combined the majority rule model with probability $p$ with the voter model with probability $1-p$ and then measure the resulting consensus times on scale-free networks. They found that that the optimized ratio to minimize consensus time is around $p=0.72$. In Refs. [38-40], Yang et al. studied the effects of heterogeneous influence of individuals on the global consensus. Each individual is assigned a weight that is proportional to the power of its degree, where the power exponent $\alpha$ is an adjustable parameter. Interestingly, it is found that there exists an optimal value of $\alpha$ leading to the shortest consensus time for scale-free networks, random networks and small-world networks.

In the voter model, an agents follows the opinion of a ran-

*Electronic address: hxyang01@gmail.com domly selected neighboring agent. In the majority rule model, an agent follows the local majority opinion. For the voter model and the majority rule model, an agents absolutely follows one opinion. However, in reality, there can be situations where an agent chooses one opinion with a stochastic probability due to bounded rationality. In this Letter, we propose a nonlinear voter model in which an agent $i$ selects one of binary opinions with the probability that is proportional to the power of the number of agents carrying this opinion among agent $i$ and its nearest neighbors. The power exponent $\alpha$ is introduced to control selective probability. Our main finding is that, there exists an optimal value of $\alpha$ for which the convergent time for consensus is minimum. This phenomenon indicates that, a suitable preference of the local majority opinion (not following it absolutely) can greatly accelerate the convergence process towards the final consensus.

\section{MODEL}

Our model is described as follows. For a given network of any topology, each node represents an agent. Initially the two opinions denoted by the values \pm 1 are randomly assigned to agents with equal probability. At each time step, agents synchronously update [41] their opinions according to the following rule. Agent $i$ will select the opinion +1 with the probability

$$
p_{+}=\frac{n_{+}^{\alpha}}{n_{+}^{\alpha}+n_{-}^{\alpha}},
$$

where $n_{+}$and $n_{-}$are the number of agents holding the opinion +1 and -1 among agent $i$ and its nearest neighbors respectively, and $\alpha$ is an adjustable parameter. Similarly, agent $i$ selects the opinion -1 with the probability $p_{-}=n_{-}^{\alpha} /\left(n_{+}^{\alpha}+n_{-}^{\alpha}\right)$, where $p_{-}=1-p_{+}$.

The parameter $\alpha$ characterizes the degree of herd effect on consensus. For $\alpha>0(<0)$, agent $i$ has a larger probability to select the local majority (minority) opinion. In the case 


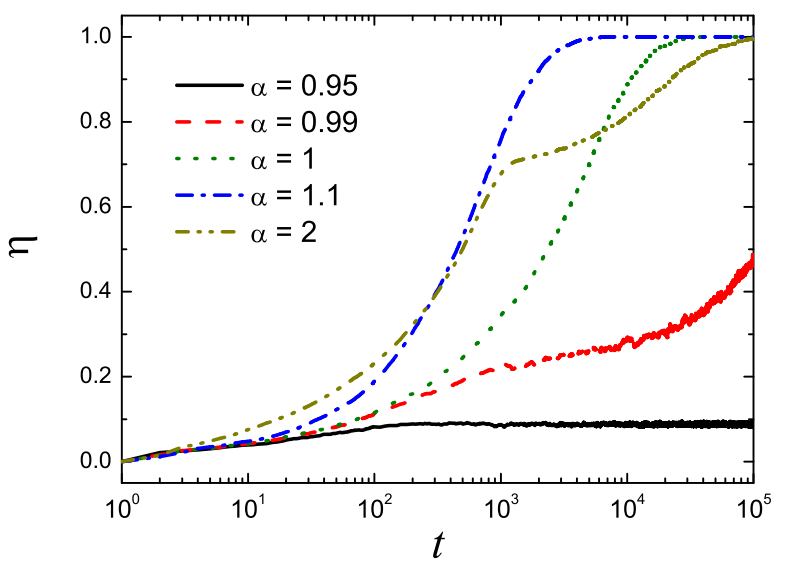

FIG. 1: (Color online.) Order parameter $\eta$ as a function of $t$ for different values of $\alpha$ on a $50 \times 50$ square lattice with periodic boundary conditions. Each data point is obtained by averaging over 2000 different realizations.

of $\alpha=0$, agent $i$ randomly selects the opinion +1 or -1 . For $\alpha=1$, our model coincides with the voter model. For $\alpha \rightarrow \infty$, agent $i$ absolutely takes the local majority opinion and our model is reduced to the model proposed in Ref. [15].

\section{RESULTS AND ANALYSIS}

We first consider a square lattice with periodic boundary conditions. Following Ref. [10], we define an order parameter $\eta$ as

$$
\eta=\frac{1}{N}\left|\sum_{i=1}^{N} \sigma_{i}\right|,
$$

where $\sigma_{i}$ is the value of node $i$ 's opinion $(+1$ or -1$)$ and $N$ is the total number of nodes of the network. In general, we have $0 \leq \eta \leq 1$, and a large value of $\eta$ indicates that one opinion in the system dominates the other. If the two opinions are equally probably, we have $\eta=0$. When global consensus is achieved so that there is only one opinion in the system, we have $\eta=1$.

Figure 1 shows the order parameter $\eta$ as a function of the time step $t$ for different values of $\alpha$. For $\alpha=1,1.1$ and 2, $\eta$ increases from 0 to 1 with $t$. For $\alpha=2.0$, initially $\eta$ increases faster than the cases of $\alpha=1.0$ and $\alpha=1.1$. Nevertheless, the system converges to the global consensus state faster for $\alpha=1.1$ than for $\alpha=2.0$. Another feature in Fig. 1 is that, for $\alpha<1$, the time required to converge to global consensus can be extremely long.

Why does the system become so difficult to reach consensus for $\alpha<1$ ? According to the mean-field approximation, opinion dynamics in the preferential-selection model can be

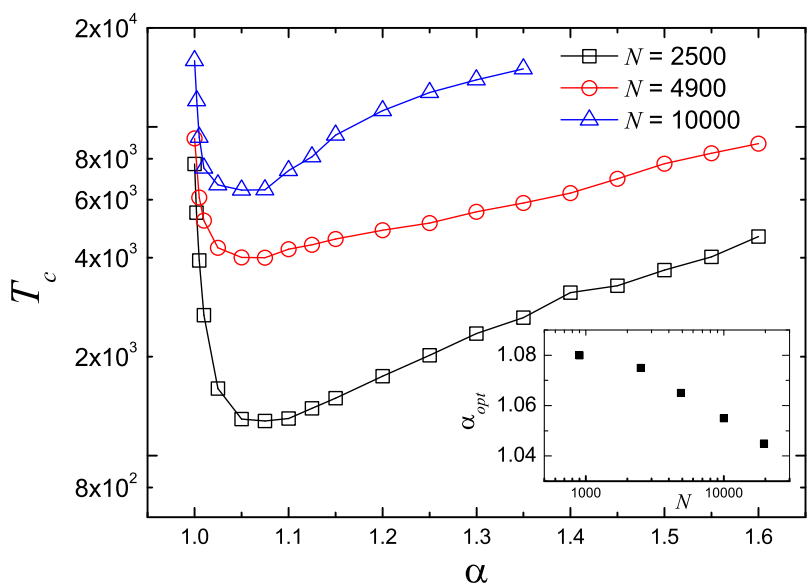

FIG. 2: Time to achieve consensus, $T_{c}$, as a function of $\alpha$ for different lattice size $N$. The inset shows the optimal value of $\alpha, \alpha_{o p t}$, as a function of $N$. Each data point is obtained by averaging over 2000 different realizations.

described as:

$$
\begin{gathered}
\frac{d \rho_{+}}{d t}=\frac{\rho_{+}^{\alpha} \rho_{-}}{\rho_{+}^{\alpha}+\rho_{-}^{\alpha}}-\frac{\rho_{-}^{\alpha} \rho_{+}}{\rho_{+}^{\alpha}+\rho_{-}^{\alpha}} \\
=\frac{\rho_{+}^{\alpha}\left(1-\rho_{+}\right)}{\rho_{+}^{\alpha}+\left(1-\rho_{+}\right)^{\alpha}}-\frac{\rho_{+}\left(1-\rho_{+}\right)^{\alpha}}{\rho_{+}^{\alpha}+\left(1-\rho_{+}\right)^{\alpha}} \\
=\frac{\rho_{+}\left(1-\rho_{+}\right)}{\rho_{+}^{\alpha}+\left(1-\rho_{+}\right)^{\alpha}}\left[\rho_{+}^{\alpha-1}-\left(1-\rho_{+}\right)^{\alpha-1}\right],
\end{gathered}
$$

where where $\rho_{+}$and $\rho_{-}$are the fractions of agents holding the opinion +1 and -1 in the total population, respectively. In the case of $\alpha<1$, for $\rho_{+}>0.5(<0.5), d \rho_{+} / d t<$ $0(>0)$. This indicates a negative feedback mechanism that prohibits the majority opinion from spreading over the whole population.

We define the consensus time $T_{c}$ as the time steps required to reach the final consensus. Figure 2 shows that $T_{c}$ as a function of $\alpha$ for different network size $N$. It can be seen that there exists an optimal value of $\alpha$, hereafter denoted by $\alpha_{o p t}$, which results in the shortest consensus time $T_{c}$. We have found that $\alpha_{\text {opt }}$ decreases as the network size $N$ increases, as shown in the inset of Fig. 2 ,

To understand the process of convergence to consensus, we study the evolution of opinion clusters. A opinion cluster is a connected component (subgraph) fully occupied by nodes holding the same opinion. Figure 3 shows the number of opinion clusters $N_{c l}$ and the normalized size of the largest cluster $S_{1}=S_{\max } / N$ as a function of $t$ for different values of $\alpha$, where $S_{\max }$ is the size of the largest cluster. We see that eventually $N_{c l}$ decreases to 1 and $S_{1}$ increases to 1 for large $t$. For small values of $\alpha$, agents do not select the local majority opinion with large probability, thus it becomes difficult for them to form large opinion clusters. For with $\alpha=1.0$, at the beginning, $N_{c l}$ decreases and $S_{1}$ increases much more slowly. For $\alpha=2.0$, initially $N_{c l}$ decreases and $S_{1}$ increases more quickly than the cases of $\alpha=1.0$ and $\alpha=1.1$. However, 

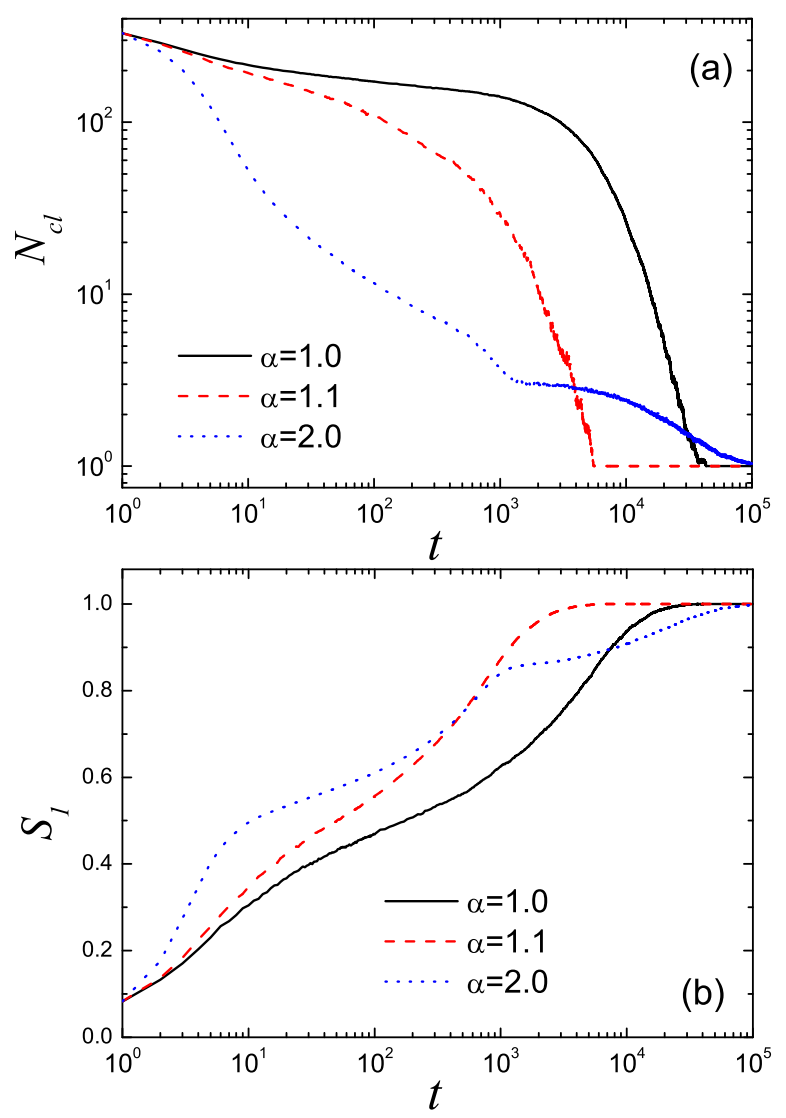

FIG. 3: (Color online.) (a) The number of opinion clusters $N_{c l}$ and (b) the normalized size of the largest cluster $S_{1}$ as a function of time $t$ for different values of $\alpha$ on a $50 \times 50$ square lattice. Each data point is obtained by averaging over 2000 different realizations.
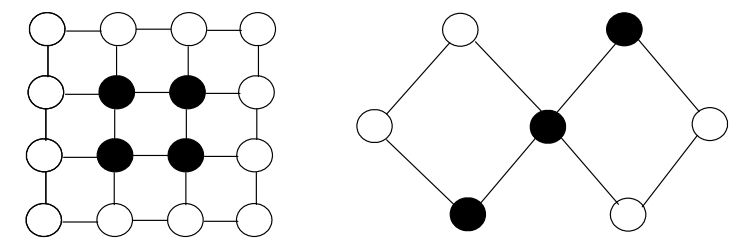

FIG. 4: Illustration of the configuration for the coexistence of two opinions for $\alpha \rightarrow \infty$.

when there are only two or three opinion clusters remained in the system, $N_{c l}$ decreases and $S_{1}$ increases very slowly for $\alpha=2.0$, indicating that the merging of different clusters becomes difficult for large values of $\alpha$. In particular, for $\alpha \rightarrow \infty$, the system cannot reach consensus in some cases. As shown in Fig. 4, two different opinions can coexist forever when $\alpha \rightarrow \infty$. For moderate values of $\alpha(\alpha=1.1)$, large opinion clusters can be formed more rapidly than for low val-
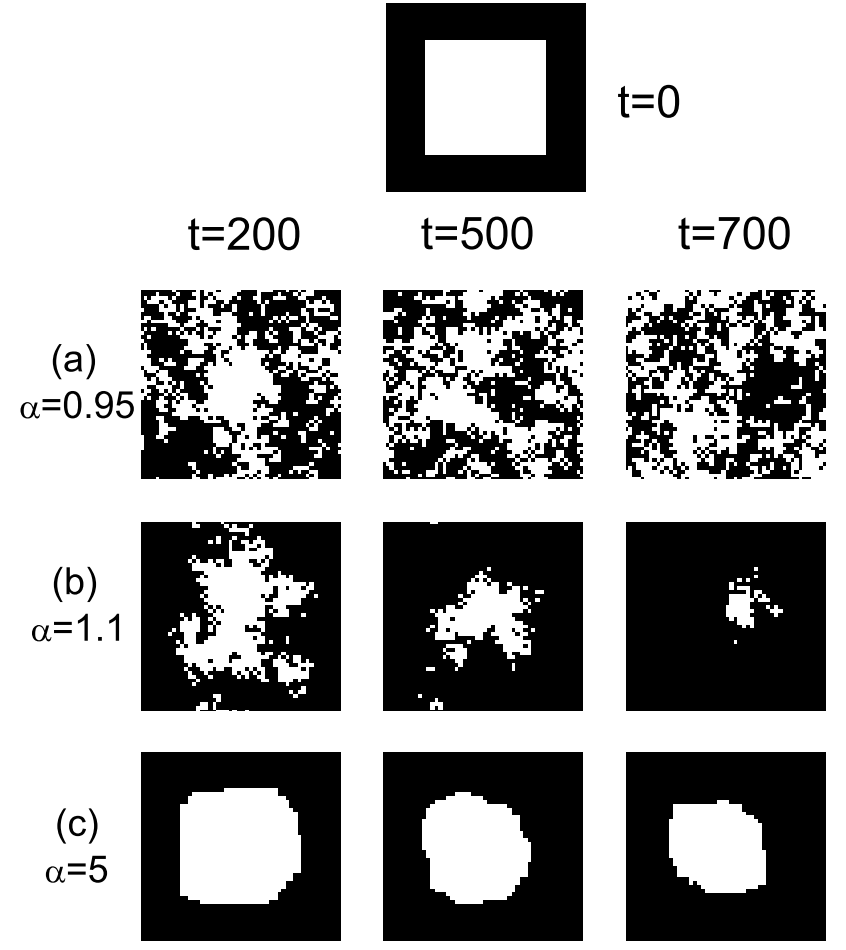

FIG. 5: Snapshots of opinion patterns on a $50 \times 50$ square lattice. Initially $(t=0)$, we set a subregion of $30 \times 30$ at the center where all agents have the opinion +1 (white), but the other nodes carry the opinion -1 (black). Initially the fraction of the opinion +1 is $\rho_{+}(0)=9 / 25=0.36$. (a) $\alpha=0.95, \rho_{+}(200)=0.41$, $\rho_{+}(500)=0.4576, \rho_{+}(700)=0.474$; (b) $\alpha=1.1, \rho_{+}(200)=$ $0.2712, \rho_{+}(500)=0.1276, \rho_{+}(700)=0.0278$; (c) $\alpha=5$, $\rho_{+}(200)=0.3232, \rho_{+}(500)=0.2428, \rho_{+}(700)=0.1916$.

ues of $\alpha(\alpha=1.0)$, and the their emergence is facilitated than the cases of larger values of $\alpha$ (e.g., $\alpha=2.0$ ) as well. Thus the convergence time becomes minimum for some moderate value of $\alpha$. It can also be noted that the evolution of $S_{1}$ for different values of $\alpha$ is similar to that of $\eta$ (see Fig. (1).

To assess how different values of $\alpha$ affect the evolution of the opinion clusters, we design a numerical procedure to investigate the evolution of spatial patterns of opinion. Specifically, in the $50 \times 50$ square lattice, initially we set a subregion of $30 \times 30$ at the center where all agents have the opinion +1 , but the other nodes carry the opinion -1 , as shown in Fig. 5. We see that for $\alpha<1$, e.g., $\alpha=0.99$, the boundary between the two opinion clusters gradually become blurred and they tend to be well-mixed with approximately equal densities. In this case, global consensus is less likely due to the difficulty to form opinion clusters. For moderate values of $\alpha$, e.g., $\alpha=1.1$, the boundaries become irregular with time but finally the +1 cluster vanishes. For high values of $\alpha$, e.g., $\alpha=5.0$, the boundaries are clear and the inside cluster shrinks but at a speed lower than that for $\alpha=1.1$.

The existence of an optimal value of $\alpha$ for which global 


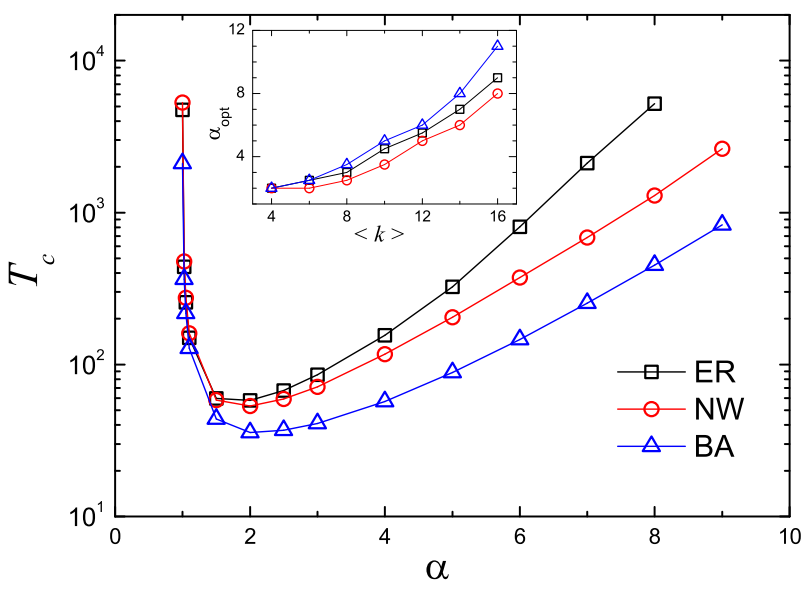

FIG. 6: (Color online.) Convergent time $T_{c}$ as a function of $\alpha$ for ER random graphs, NW small-world networks and BA scale-free networks. The average connectivities of ER, NW and BA networks are 4. The inset shows the value of $\alpha_{\text {opt }}$ as a function of the average connectivity $\langle k\rangle$ for the three types of networks. The network size is $N=3000$. Each data point is obtained by averaging over 100 different network realizations with 20 runs for each realization.

consensus can be achieved rapidly is not restricted to the square-lattice structure. In fact, we have observed a similar behavior for complex networks including Erdös-Rényi random graphs (ER) [42], Newman-Watts small-world networks (NW) [43] and Barabási-Albert scale-free networks (BA) [44]. We also observe that, the optimal value of $\alpha$ tends to increase with the average connectivity $\langle k\rangle$ (see the inset of Fig. 6). From Fig. 6, one can see that the topological structure affects the convergent time $T_{c}$. Among the three complex net- works, $T_{c}$ for scale-free networks is shortest when $\langle k\rangle$ and $\alpha$ is fixed.

\section{CONCLUSION}

In summary, we have proposed a nonlinear voter model to study the convergence to global consensus on lattices and complex networks. An agent $i$ selects one of binary opinions with the probability that is proportional to the power of the number of agents carrying this opinion among agent $i$ and its nearest neighbors. The power exponent $\alpha$ is introduced to govern selective probability. It is found that there exists an optimal value of $\alpha$ leading to the shortest convergent time. We have explained such phenomenon in terms of the evolution of the opinion clusters. For too small values of $\alpha$, the formation of big opinion clusters is slow. For very high values of $\alpha$, the merging of different opinion clusters becomes difficult. Taken together, the shortest convergent time can be realized at moderate values of $\alpha$. Our results indicate that, the consensus would be quickly reached if agents suitably follow the local majority opinion.

\section{Acknowledgments}

HXY and BHW were supported by the National Basic Research Program of China (973 Program No. 2006CB705500); the National Important Research Project (Grant No. 91024026); the National Natural Science Foundation of China (Grant Nos. 11005001, 10975126 and 10635040), the Specialized Research Fund for the Doctoral Program of Higher Education of China (Grant No. 20093402110032). WXW and YCL were supported by AFOSR under Grant No. FA955010-1-0083.
[1] C. Castellano, S. Fortunato, and V. Loreto, Rev. Mod. Phys. 81 (2009) 591.

[2] T. M. Liggett, Stochastic Interacting Systems: Contact, Voter, and Exclusion Processes (Springer, Berlin, 1999).

[3] V. Sood and S. Redner, Phys. Rev. Lett. 94 (2005) 178701.

[4] S. Galam, Eur. Phys. J. B 25 (2002) 403.

[5] P. L. Krapivsky and S. Redner, Phys. Rev. Lett. 90 (2003) 238701.

[6] G. Duffuant, F. Amblard, G. Weisbuch, and T. Faure, J. Artif. Soc. Soc. Simul. 5 (2002) 4.

[7] A. Nowak, M. Kuś, J. Urbaniak, and T. Zarycki, Physica A 287 (2000) 613.

[8] R. Albert and A.-L. Barabási, Rev. Mod. Phys. 74 (2002) 47.

[9] M. J. de Oliveira, J. Stat. Phys. 66 (1992) 273.

[10] A. D. Sánchez, J. M. López, and M. A. Rodríguez, Phys. Rev. Lett. 88 (2002) 048701.

[11] P. Chen and S. Redner, Phys. Rev. E 71 (2005) 036101.

[12] P.-P. Li, D.-F. Zheng, and P. M. Hui, Phys. Rev. E 73 (2006) 056128.

[13] F. Vazquez, V. M. Eguíluz, and M. S. Miguel, Phys. Rev. Lett. 100 (2008) 108702.

[14] C. Nardini, B. Kozma, and A. Barrat, Phys. Rev. Lett. 100 (2008) 158701.
[15] J. Shao, S. Havlin, and H. E. Stanley, Phys. Rev. Lett. 103 (2009) 018701.

[16] Z.-X. Wu and P. Holme, Phys. Rev. E 81 (2010) 011133.

[17] C. M. Schneider-Mizell and L. M. Sander, J. Stat. Phys. 136 (2009) 59.

[18] Z.-X. Wu and P. Holme, Phys. Rev. E 82 (2010) 022102.

[19] S. Galam, Physica A 333 (2004) 453.

[20] C. Castellano, V. Loreto, A. Barrat, F. Cecconi, and D. Parisi, Phys. Rev. E 71 (2005) 066107.

[21] S. Galam, Phys. Rev. E 71 (2005) 046123.

[22] K. Suchecki, V. M. Eguíluz, and M. S. Miguel, Phys. Rev. E 72 (2005) 036132.

[23] L. F. C. Pereira and F. G. B. Moreira, Phys. Rev. E 71 (2005) 016123.

[24] P. Holme and M. E. J. Newman, Phys. Rev. E 74 (2006) 056108.

[25] M. C. González, A. O. Sousa, and H. J. Herrmann, Eur. Phys. J. B 49 (2006) 253.

[26] S. Gil and D. H. Zanette, Phys. Lett. A 356 (2006) 89.

[27] R. Lambiotte, Europhys. Lett. 78 (2007) 68002.

[28] R. Lambiotte, M. Ausloos, and J. A. Hołyst, Phys. Rev. E 75 (2007) 030101(R).

[29] V. Sood, T. Antal, and S. Redner, Phys. Rev. E 77 (2008) 041121. 
[30] H.-U. Stark, C. J. Tessone, and F. Schweitzer, Phys. Rev. Lett. 101 (2008) 018701.

[31] B. Kozma and A. Barrat, Phys. Rev. E 77 (2008) 016102.

[32] F. Vazquez and C. Lopez, Phys. Rev. E 78 (2008) 061127.

[33] C. Castellano, M. A. Muñoz, and R. Pastor-Satorras, Phys. Rev. E 80 (2009) 041129.

[34] F. Vazquez, X Castelló, and M. San Miguel, J. Stat. Mech. (2010) P04007.

[35] N. Masuda, N. Gibert, and S. Redner, Phys. Rev. E 82 (2010) 010103(R).

[36] C. Castellano and R. Pastor-Satorras, Phys. Rev. E 83 (2011) 016113.
[37] J.-S. Yang, I.-M. Kim, and W. Kwak, EPL 88 (2009) 20009.

[38] H.-X. Yang, Z.-X. Wu, C. Zhou, T. Zhou, and B.-H. Wang, Phys. Rev. E 80 (2009) 046108.

[39] Y.-T. Lin, H.-X. Yang, Z.-H. Rong, and B.-H. Wang, Int. J. Mod. Phys. C 21 (2010) 1011.

[40] H.-X. Yang and B.-H. Wang, Physics Procedia 3 (2010) 1859.

[41] We have checked that the qualitative results do not change when asynchronous update is used.

[42] P. Erdős and A. Rényi, Publ. Math. (Debrecen) 6 (1959) 290.

[43] M. E. J. Newman and D. J. Watts, Phys. Lett. A 263 (1999) 341.

[44] A.-L. Barabasi and R. Albert, Science 286 (1999) 509. 